\title{
FORMAS COMBINANTES: UN ESTUDIO SOBRE LOS PROCESOS MORFOLÓGICOS DE TRUNCAMIENTO EN ESPAÑOL
}

\author{
Mónica Cantero
}

\begin{abstract}
RESUMEN
Este trabajo propone considerar dos puntos importantes en la morfología del español. Primero, aportar una descripción adecuada para una serie de palabras que parecen estar entre la afijación y la composición. Segundo, gracias a esta descripción, señalar la relevancia que el proceso morfológico de truncamiento y sus unidades (formas combinantes) tienen en la creación de nuevas palabras en el léxico español. Asimismo, este proceso morfológico de truncamiento es un claro reflejo de las motivaciones pragmáticas empleadas por el hablante-oyente de una lengua con la finalidad de manipular procesos morfológicos y crear de esta manera nuevo léxico y connotaciones lingüísticas en la lengua. Palabras clave: morfología, pragmática, truncamiento, formas combinantes.
\end{abstract}

\begin{abstract}
This work will consider two important points in the Spanish morphology. First, to contribute an adapted description for a series of words that appears to be between affixation and composition. Second, thanks to this description, it will show the relevance that the morphologic process of secretion and its units (combining forms) have in the creation of new words in the Spanish lexicon. Also, this morphological process of secretion is a clear reflection of the pragmatic motivations used by the speakerlistener of a language with the purpose of manipulating morphologic processes and creating from this manner a new lexicon and linguistic connotations in the language.
\end{abstract}

Key words: morphology, pragmatics, secretion, combining forms.

\section{Introducción}

El acercamiento a la investigación de la estructura de las palabras que exploramos en este trabajo (ver también Cantero 2001) representa la convergencia de dos diferentes avenidas de investigación. La primera es la teoría morfológica y léxica (Aronoff 1976, Scalise 1984) y la segunda proviene del estudio de los efectos y significados pragmáticos generales que resultan de la aplicación de las reglas morfológicas (Dressler y Barbaressi 1987).

Dr. Mónica Cantero. Spanish Department, Drew University.

Madison, New Jersey, U.S.A.

Correo electrónico: mcantero@ drew.edu 
El objetivo de este trabajo es llevar a cabo un estudio de las motivaciones pragmáticas de las estrategias morfológicas productivas de los procesos de truncamiento, en particular (Warren 1990) las empleadas por el usuario de la lengua con el fin de crear / reflejar de la mejor forma posible sus intenciones comunicativas cuando usa las reglas de formación de palabras para manipular un nuevo concepto o significado en la lengua. Es un reflejo de los procesos mentales, los cuales determinan cómo un usuario de la lengua es capaz de combinar forma y significado en sistemas de reglas morfológicas.

En el marco teórico propuesto por Dressler y Barbaressi (1987) para establecer las bases que constituyen el campo de la morfopragmática, se plantea que existen ciertos principios universales pragmáticos en la morfología y que hay una relación patente entre las reglas morfológicas y sus intérpretes, así como también en la interpretación de los resultados de una regla de formación de palabras (RFP) posible o real (Aronoff 1976). En nuestro análisis de los datos recogidos examinaremos, desde esta perspectiva, una relación que establezca la conexión creada entre el resultado de una RFP y la interpretación recibida por el oyente, así como el significado implicado, creado por el hablante en el proceso de aplicación de las RFP.

\section{Caracterización del corpus}

En español, como en inglés, existen unas palabras que parecen estar entre la composición y la afijación. Nos referimos a palabras como teléfono, fonología, polideportivo, multinacional, etc. En la mayoría de estos casos, parte del elemento es una forma clásica (latina o griega). Algunos autores han tratado estos casos como compuestos neoclásicos. La pregunta que planteamos es cómo analizar morfológicamente formas de la clase fono, sono, poli, multi. Estas son utilizadas en la creación léxica como elementos productivos de la lengua. Aisladas, son formas posibles pero no existentes y presentan la peculiaridad de que no pueden ser tratadas simplemente como afijos, ya que no existe ningún afijo que pueda estar en posición prefijada unas veces y en otros contextos en posición sufijada: [fonología] [teléfono].

Scalise (1984) resuelve este problema considerando tales unidades como temas y no como afijos, lo que le permite tratar estas formas de igual manera tanto si aparecen en posición inicial como en posición final. Es un enfoque que le posibilita tratar estas formas lingüísticas como compuestos. Como consecuencia de este planteamiento, se desprende la necesidad de incluir en el lexicón una subparte que dé cuenta de los elementos cultos. Scalise aboga por esta solución para resolver el conflicto de tratamiento de estas formas clásicas.

Uno de los problemas de dicho planteamiento es que estos temas no pueden funcionar solos y no poseen rasgos categoriales. En la siguiente muestra observaremos que estas formas cultas han sido derivadas mediante un afijo [-ic] y flexionadas [-o], lo cual supone la existencia de ciertos rasgos sintáctico-semánticos que permiten el uso de tales formas como bases en el proceso derivativo. Sin este particular contenido informativo, las RFP no hubieran podido derivar dicha forma: 


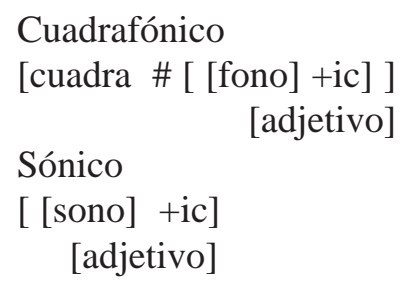

\section{Presentación del modelo morfológico elegido y descripción de los procesos de creación de nuevas palabras}

Warren (1990: 115) aporta una adecuada explicación descriptiva de estos morfemas que parecen afijos para el inglés. La clasificación que discute es la de considerar dichos elementos como FORMAS COMBINANTES (combining forms) que pueden aparecer tanto en posición final como en posición inicial. En esta investigación, hemos adaptado dicha terminología para desarrollar una explicación satisfactoria del comportamiento morfológico de tales elementos en español.

Las formas combinantes finales e iniciales pueden ser subdivididas en dos grupos:

1. elementos que son variantes alomórficas de alguna otra palabra: astro- (del latín astrum), -dromo (del griego dromos).

2. elementos que representan parte de otras palabras: ciber-, -ólico. Estos elementos pueden ser formados tanto a partir de palabras existentes en español como de palabras procedentes de otra lengua (en la mayoría de los casos, del inglés).

Relativo a la diferencia de comportamiento de las formas iniciales en contraste con las finales, Warren (1990: 115) afirma que "initial combining forms differ from final ones in that the former have a characteristic phonetic shape, whereas the latter are amorphous in this respect”. La subdivisión propuesta recoge no solamente préstamos clásicos adaptados fonéticamente (en el estudio de Warren, al inglés), sino que también encontramos elementos no clásicos y nuevos morfemas.

Warren centra el foco de su estudio en observar de qué manera pueden ser creados estos nuevos morfemas con significado innovador. En concreto, propone que es a través del PROCESO DE TRUNCAMIENTO. Una de las características de este proceso es que implica reajustar los elementos semánticos. Este reajuste significa que ciertos elementos de tipo semántico que no habían sido asociados con ninguna parte de la forma de la palabra pasan a ser conectados a una determinada sección de dicha palabra. Warren (1990: 117) explica la naturaleza de este proceso con el ejemplo de la forma combinante -(a)holic: "-(a)holic, I claimed, is a content morpheme meaning "person addicted to", which is a meaning we derive from alcoholic, although we do not associated "person addicted to" with the phoneme sequence -holic in alcoholic".

Consideremos como ejemplo el caso de eróscopo (de la revista Cosmopolitan, agosto 2001) que encontramos en español. Es poco plausible pensar que la gente confundiera [ero-] por un morfema que significa 'erótica' y [-óscopo] por un morfema con el significado de 'tipo 
de formato comunicativo dedicado a la predicción del futuro'. En esta investigación se sugiere que el hablante que crea la palabra eróscopo no toma [ero-] por un morfema, sino que lo convierte en uno. En consecuencia, utilizando este recurso de truncamiento de nuevos morfemas, se mantiene la parte de la palabra que el hablante juzga necesaria y efectiva para desencadenar las asociaciones correctas y tener las características fonológicas adecuadas de morfema para que sea un componente válido de la palabra. El resto es, simplemente, desechado por el hablante. Se reemplaza de esta manera la parte descartada por otro morfema con contenido, el cual añade finalmente el rasgo específico de significado con las connotaciones deseadas de significado que el hablante se ha propuesto crear.

Podría decirse que eróscopo ha sido formado truncando [-óscopo] de horóscopo y [ero-] de erótica, lo que provoca en el oyente las siguientes asociaciones: "Columna informativa diseñada con la intención de proveer al lector con la bendición astral en el terreno amoroso".

\section{Tablas de clasificación de los elementos combinantes: elementos combi- nantes en posición inicial}

Tabla 1. Elementos combinantes de origen clásico

\begin{tabular}{lll} 
"prefijos" & combinación & palabra que sirve de modelo \\
\hline $\begin{array}{ll}\text { astro- } \\
\text { audio- }\end{array}$ & astrofísica & lat. astrum \\
auto- & audioconvertidor & lat. aud- \\
biblio- & autoproducir & gr. autos \\
bio- & biblioplaza & gr. biblioteca \\
& biosensor, biodegradable, & gr. bios \\
electro- & biomolécula, biotecnología & \\
etno- & electromagnético & lat. electricus \\
hetero- & etnomuseo & gr. ethnos \\
hidro- & heterosexual & gr. heteros \\
hiper & hidromasaje & gr. hydor \\
lito- & hipermercado, hiperespacio & gr. hiper \\
maxi- & litografía & gr. lithos \\
mega- & maxisingle & lat. maximus \\
micro- & megahercios & gr. megas \\
mini- & microprocesador, microgravedad & gr. micros \\
multi- & minidisc, minicadena & lat. minimus \\
neuro- & multiinstrumentista, multiplexor & lat. multus \\
orto- & neurociencia & gr. neuron \\
paleo- & ortodoncia, ortorexia & gr. recto, correcto \\
petro- & paleozoología & gr. palaiós \\
poly- & petroquímica, petrodólar & lat. petra \\
psico- & polideportivo & gr. polys \\
sono- & psicodelia & gr. psyche \\
tecno- & subsónico, sonograma & lat. sonus \\
& tecnopop & gr. techne \\
& &
\end{tabular}


tele-

telecomedia, telecupón, teletienda, gr. telos teletexto, telebanco

Tabla 2. Elementos combinantes que son parte de otras palabras

$\begin{array}{lll}\text { anarco- } & \text { anarcopunk, anarcosindicalistas } & \text { anarquía } \\ \text { ciber- } & \text { ciberpunk, ciberdélicos } & \text { cibernético } \\ \text { docu- } & \text { docudrama } & \text { documental } \\ \text { eco- } & \text { ecosistema } & \text { ecología } \\ \text { ero(s)- } & \text { eróscopo } & \text { erótica } \\ \text { Euro- } & \text { eurocopa, euromoneda } & \text { Europa } \\ \text { narco- } & \text { narcotráfico, narcodólar } & \text { narcóticos }\end{array}$

\section{Elementos combinantes en posición final}

Tabla 3. Elementos combinantes finales que son de origen clásico

\begin{tabular}{lll} 
"sufijo" & combinación & palabra que sirve de modelo \\
\hline -delia & psicodelia & gr. deloyn, délos \\
-dromo & velódromo, canódromo & gr. dromos \\
-fobia & ciberfobia & gr. phobia \\
-grama & organigrama, electrocardiograma & gr. gramma \\
-(o)logo & bluesólogos & gr. logos \\
-manía & calcomanía & gr. manía \\
-(o)teca & funkoteca & gr. biblioteca
\end{tabular}

Tabla 4. Elementos combinantes finales que son parte de otras palabras

-(a)holic
-asta
-delia
-delicos
-scopo
-matico
-rexia
-rrea
-zine

trabajólico

videasta

funkadelia

ciberdélicos

eróscopo

hidromático

ortorexia

seborrea, verborrea

fanzine, ciberzine alcohólico

cineasta

psicodelia

psicodélicos

horóscopo

automático

anorexia

dia /rrea (dos raíces griegas)

magazine

\section{Comportamiento de las formas combinantes y el proceso morfológico de truncamiento}

En las tablas 3 y 4 encontramos ejemplos de formas truncadas, pero no todas estas formas combinantes han sido creadas mediante el proceso de truncamiento; algunas de ellas son abreviaciones. Encontramos abreviaciones de las formas iniciales particularmente en la 
tabla 2. Una posible excepción en este grupo sería ciber-, docu-, narco- (ciber- expresa el significado de 'máquina-computador', docu- expresa 'informativo', narco- expresa 'sustancia ilegal'). Dicho proceso es posible en estas formas mediante una transferencia semántica que no estaba asociada a ninguna parte concreta de la palabra: cibernética, documental, narcóticos.

El proceso de truncamiento se caracteriza por mantener sólo ciertos rasgos semánticos; es una unidad incompleta y el hablante tiene que interpretar y descartar los rasgos semánticos pertinentes. Los elementos semánticos que se han descartado requieren ser reemplazados. En el proceso de abreviación todos los rasgos semánticos se mantienen aunque la unidad lingüística esté acortada; el resultado es una unidad completa.

Truncamiento y abreviación son procesos productivos de creación y explotación de referencias léxicas, que muestran un conocimiento léxico compartido entre hablantes y oyentes de una lengua. El mismo fenómeno ocurre tanto en inglés como en español. Por ejemplo, si necesitamos una palabra para la noción de 'persona adicta al trabajo' que se parece a la noción de 'persona adicta al alcohol', a excepción de la noción semántica de 'alcohol', usamos esta última noción manteniendo la parte de la palabra que proporciona la idea de 'persona adicta a'. Descartamos el resto de la unidad lingüística e indicamos el cambio de modificación semántica reemplazando el elemento que hemos descartado con la palabra que indica 'trabajo'. El resultado es trabajólico en español y workoholic en inglés. El morfema -(a)holic es particularmente productivo en lengua inglesa, ya que existe también spendaholic, forma combinante creada usando este proceso de truncamiento. Sobre spendaholic, Warren (1990: 121) comenta que este término sugiere más que simplemente 'persona adicta a': "the behavior of a spendaholic is an important social phenomenon. It often results from the lack of security. It sometimes afflicts young people and often those that have been deserted by their spouse. It is not restricted to those two typical groups, of course".

Consideremos el caso de la introducción de la forma -zine en el español reciente peninsular. -Zine, que sugerimos es una forma combinante final, es una parte lingüística en la palabra inglesa magazine, que significa 'revista'. La combinación y el uso de este segmento como otros morfemas con contenido, como lo demuestra nuestro ejemplo fanzine, es relativamente reciente (recogimos evidencias de una primera documentación en el año 1989, en revistas de música publicadas en España) y productivo, dado que su penetración en la lengua ha sido asentada favorablemente mediante el uso en contexto de este morfema por los usuarios del lenguaje. Una de las características de esta unidad lingüística es que puede funcionar como morfema ligado, parte incorporada en la forma de la palabra — consideremos el ejemplo fanzine, y también otro faszine- o puede funcionar como morfema libre \#zine en los contextos documentados Zine de R'n'R, Zine la Chica de la Montaña n.2. En estas situaciones, encontramos la forma Zine como núcleo categorial sustantivo de un sintagma nominal de tipo $[\mathrm{N}+\mathrm{de}+\mathrm{Adj}]$. El análisis morfológico de fanzine refleja la estructura de formación siguiente:

\section{magazine}

- Proceso de truncamiento: [maga] + [zine]

- Modificación y reajuste de los elementos semánticos. Elección de la referencia semántica —efecto locutivo- que deseamos conllevar o conectar a una determinada sección de la palabra o forma. [Zine]: "formato de revista especializada". 
- Búsqueda de un morfema con contenido que aporte los nuevos rasgos de modificación semántica que deseamos ejecutar en la nueva creación. [fan]: 'partidarios de un tipo de creencia, gusto, moda, etc.'

$[$ fan $]+[$ zine $]$

- Reemplazo de la unidad descartada [maga] por el nuevo morfema con contenido

- Forma final resultante. Significado "revista cuyo contenido tiene que ver exclusivamente con el mundo de los fans". Fanzine.

Podemos desprender de este ejemplo que la formación de ciertas formas combinantes, como por ejemplo la reciente introducción de la forma -zine, puede ser una herramienta poderosa de creación de nuevos morfemas en una lengua.

Warren (1990: 120) afirma que construir el significado de las palabras que contienen formas combinantes formuladas mediante el proceso de truncamiento requiere más proceso mental del que en un principio podemos suponer. Una de las razones es que el significado relacional no permanece en la palabra resultante y, consecuentemente, debe ser construido desde un principio. El éxito o fracaso semántico de la forma resultante de un proceso morfológico - derivación o composición - muchas veces depende de un conocimiento extra-lingüístico asumido y compartido entre los hablantes, que sería precisado por los morfemas que estemos manipulando en cuestión.

Entender el resultado final de una forma combinante que ha sido obtenida mediante el proceso de truncamiento precisa de dos niveles: un requisito es reconocer la forma modelo que ha sido usada (forma, significado y referentes); el segundo aspecto es poder reajustar los rasgos semánticos y añadir el nuevo morfema con contenido semántico preciso requerido con el fin de obtener el resultado óptimo final. Una conjunción de tres focos diferentes entra en relación durante este proceso: adición del morfema con contenido, nuestro conocimiento intuitivo de la palabra modelo y la nueva combinación.

De todos los factores que intuimos sobre el referente que tomamos como modelo, escogemos algunos rasgos de significado. Por ejemplo, al seleccionar los rasgos semánticos de -rrea en verborrea o seborrea, seleccionaremos 'flujo excesivo de' como rasgo semántico fijo. Es una intención que deseamos conllevar en la forma resultante. Por lo tanto, cabe mencionar que se produce un acto ilocutivo paralelo a la selección de rasgos semánticos que refleja una serie de intenciones comunicativas determinadas. El hablante intenta conseguir un propósito específico. Esta observación ofrece un ángulo de perspectiva sobre una dimensión diferente del sentido del uso de los procesos morfológicos.

Determinadas formas combinantes pueden ser ricas en connotaciones. Por ejemplo, - rexia es una forma combinante en ortorexia 'obsesión patológica por la comida sana, hasta el punto extremo de sufrir una dependencia psicológica'. Es una nueva palabra que procede del griego orto- 'correcto, recto' y del truncamiento de la palabra anorexia. Esta nueva palabra proporciona significado y forma, mediante el proceso de truncamiento, a un nuevo tipo de enfermedad que surge en estos momentos en nuestra sociedad. Ortorexia da forma a la idea de 'obsesión por la correcta calidad de la comida ingerida'. El opuesto de este concepto es, obviamente, 'anorexia' en el que la obsesión se centra en la cantidad de la comida ingerida. Podemos decir que [-rexia] se ha convertido en una forma combinante que revela un significado y uso morfológico en el contexto de 'trastorno alimentario'. 
Otros ejemplos interesantes que demuestran la productividad y justifican el uso de esta estrategia morfológica nos los brindan las palabras funkoteca y biblioplaza. La primera palabra, funkoteca, está construida sobre la base [funk] más la forma combinante -teca, a imitación de la palabra biblioteca, ya que -teca es un formante que se utiliza en palabras como hemeroteca, discoteca, cinemateca. El resultado final funkoteca es una palabra con el significado de 'lugar donde se guarda (deposita, colecciona) música funk'.

Por otra parte, la palabra biblioplaza se forma mediante el truncamiento de biblio- de la palabra biblioteca, con la connotación de 'libros', que se añade a la palabra plaza. El resultado final, biblioplaza, es una nueva palabra con el significado 'biblioteca al aire libre o lugar exterior donde se puede realizar la consulta de libros'.

La formación de estos nuevos conceptos léxicos justifica el uso de la técnica de truncamiento. Mediante este recurso morfológico, el hablante mantiene o corta de la palabra de origen tanto como crea que es necesario con el propósito de provocar unas asociaciones nuevas y correctas y tener las características fonológicas adecuadas de morfema, para que sea un componente válido de la palabra. El resto de la palabra es, como hemos visto, abandonado por el hablante.

\section{Conclusión}

La posición defendida por Warren (1990) sobre cómo clasificar las formas combinantes (en raíces o afijos) se resume en considerar que estas son diferentes a los afijos en el sentido de que son un conjunto posiblemente abierto de elementos del diccionario, mientras que los afijos son un conjunto cerrado de elementos que han sido sometidos a un proceso de gramaticalización. Las formas combinantes no están excluidas de experimentar también este proceso, por lo que podemos encontrar morfemas que, desde un punto de vista diacrónico, pueden ser consideradas como formas combinantes, pero que desde un punto de vista sincrónico son afijos (pseudo-, neo-). Su clasificación puede ser de carácter arbitrario en algunos casos individuales.

Podemos adoptar la misma tipología clasificatoria para el español. Las formas combinantes son variantes incompletas o segmentos de palabras. Deben combinarse con otras unidades para poder formar una unidad completa. En el español, deberíamos añadir que existe la posibilidad de que en algunos casos las formas combinantes iniciales tengan rasgos sintácticos y semánticos que las posibiliten a actuar de base en un proceso morfológico derivacional. Por ejemplo, la forma combinante inicial sono- es una base para formar sónico:

$$
\begin{gathered}
{[[\text { sono }]+\mathrm{ic}]} \\
\text { Nombre } \\
{[[\text { sonic }]+\text { o/a }]} \\
\text { Adjetivo }
\end{gathered}
$$

También encontramos esta forma prefijada con sub-:

[sónico] [sub + [sónico]] -ic es un sufijo que forma adjetivos a partir de bases nominales. 
Aronoff (1976: 21) alega que solamente nombres, verbos, adjetivos y adverbios pueden ser el producto de la formación de palabras y que solamente estas clases pueden ser usadas como bases en la formación de derivativos. La primera parte de esta afirmación puede ser cierta, pero los ejemplos presentan evidencia de que esta clase de elementos combinantes clásicos iniciales pueden ser usada como base, en formas establecidas como sónico, subsónico, cuadrafónico, telefónico, etc.

Creemos que este marco teórico de formas combinantes ofrece un marco adecuado para explicar la formación y las características semánticas de unidades como fanzine o eurodiputado. Es posible aplicar el término de formas combinantes tanto a los morfemas de origen clásico como a formas que han sido conseguidas mediante un proceso de truncamiento. Tal y como afirma Warren (1990), restringir el término de formas combinantes sólo a los elementos iniciales es injustificado, aunque hay diferencias definitivas entre las formas iniciales y las finales. La diferencia entre formas combinantes y afijos estriba en considerar que los afijos pertenecen a una clase cerrada de elementos y las formas combinantes son un conjunto abierto de elementos que pueden formarse en un momento. Una de las ventajas de utilizar formas combinantes para crear nuevas unidades lingüísticas es que no tenemos la restricción de utilizar sólo formas morfemáticas existentes. Esta estrategia demuestra una intención y efecto perlocutivo por parte del hablante de comunicar en la palabra resultante una determinada impresión, que desemboque en una específica reacción en el oyente.

Incluir este proceso morfológico nuevo de formación léxica utilizando las unidades que llamamos formas combinantes en los parámetros de la morfología española, y en particular dentro de este marco de investigación, se justifica porque la evidencia de las muestras analizadas marca significativamente la relación que existe entre cómo los usuarios conocen y procesan la información morfológica, que es parte esencial de la unión forma-significado, es decir, la formación de significado y la formación de palabras (Bybee 1985).

\section{Bibliografía}

Aronoff, Mark. 1976. Word Formation in Generative Grammar. Cambridge, Mass: MIT Press.

1994. Morphology by itself: stems and inflectional classes. Cambridge: MIT Press.

Bybee, Joan L. 1985. Morphology. A study of the relation between meaning and form. Amsterdam/Philadelphia: John Benjamins Publishing Company.

Cantero, M. 2001. La morfopragmática del español. Muenchen: Lincom Europa.

Dressler Wolfgang U. y Lavinia M. Barbaresi. 1987. “Elements of Morphopragmatics”. Paper presented at the International Pragmatics Conference, Antwerp. 
Scalise, Sergio. 1984. Generative Morphology. Dordrecht: Foris Publications.

Warren, Beatrice. 1990. "The Importance of Combining Forms". Contemporary Morphology. Berlin-NY: Mouton de Gruyter. 